activation lead to multisystem tissue damage. Plasmacytoid dendritc cells ( $\mathrm{pDCs}$ ) play a central role in the pathogenesis of SLE through dysregulated type I IFN production, together with activated myeloid DCs (mDCs), amplifying vicious spiral of autoimmune disorders(1). Therefore, control of the aberrant DC activation may provide an alternative treatment strategy against SLE.

Objectives: Mycophenolate mofetil (MMF), which has been used to treat lupus nephritis, specifically blocks proliferation of $B$ and $T$ lymphocytes by inhibition of inosine-5-monophosphate dehydrogenase (IMPDH). In addition, although there is evidence indicating the immunosuppressive effects of MMF on human monocyte-derived dendritic cells(2.3), there are no reports showing its effects on human blood DC subsets. Here we focused on the effects of MMF on the functions of the blood pDCs and mDCs.

Methods: We isolated human blood DCs from healthy donors using cell sorting(4) and examined the function of mycophenolic acid (MPA), which is metabolic products of MMF, on DC subsets in response to TLR-ligands and serum from patients with active SLE. Written informed consent was obtained from all healthy adult donors and SLE patients.

Results: We found that therapeutic plasma concentration range of MPA down-regulated expression of CD40, CD80 and CD86 dose-dependently on $\mathrm{mDCs}$ and $\mathrm{pDCs}$ without inducing apoptosis, in response to R848(TLR7/8 agonist) and CpG2216(TLR9 agonist), respectively. Of note, MPA profoundly suppressed IL-12 production and STAT4 expression in the mDCs and IFN-a production and IRF7 expression in the pDCs(Fig 1). We also obserbed inhibition of nuclear translocation of IRF-7 in pDCs treated with MPA by confocal microscopy(Fig 2). Furthermore, we identified that MPA had an inhibitory effect on SLE serum-induced IFN-a production by human PBMCs.

Conclusion: Our data suggest that MMF can drive a wedge into the vicious spiral of autoimmune disorders through regulating the function of not only lymphocyte but also DC subsets. Thus, we unveiled a part mechanism of the therapeutic ability of MMF against SLE.

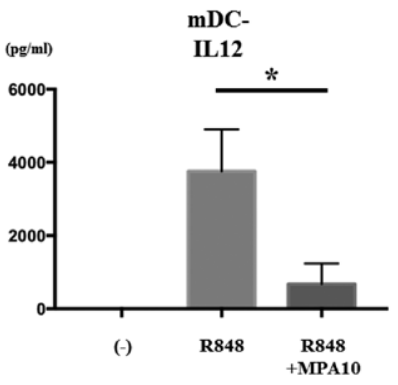

Fig2

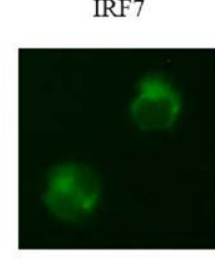

$\mathrm{CpG}$

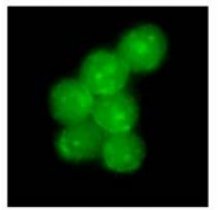

$\mathrm{CpG}$

add MPA

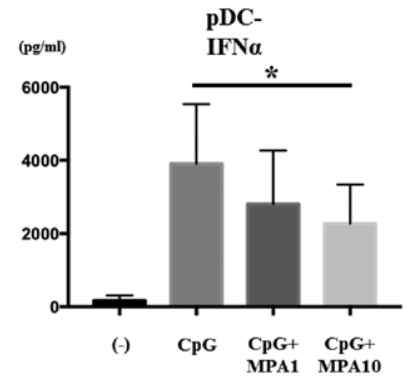

MPA: $\mu \mathrm{M}$
DAPI
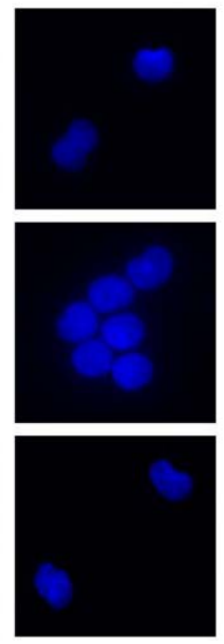

merge
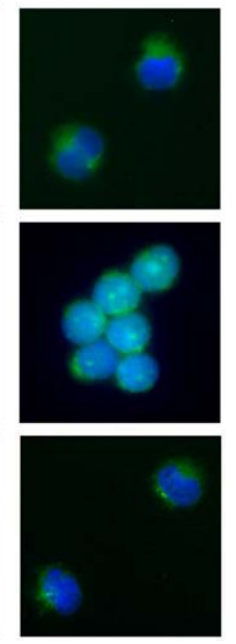

References:

[1] A Plasmacytoid Dendritic Cells-Type I Interferon Axis is Critically Implicated in the Pathogenesis of Systemic Lupus Erythematosus. Int. J. Mol. Sci. 2015: 16, 14158-14170.
[2] Mycophenolate mofetil inhibits differentiation, maturation and allostimulatory function of human monocyte-derived dendritic cells. Clin Exp Immunol. 2003;134:63-69.

[3] Mycophenolic acid inhibits maturation and function of human dendritic cells and B cells. Human Immunol. 2009;70: 692-700.

[4] Miyamoto et al. Arthritis Research \& Therapy 2010, 12:R87.

Disclosure of Interests: None declared

DOI: 10.1136/annrheumdis-2020-eular.3199

\section{AB0142 ANTIPHOSPHOLIPID SYNDROME AND ATHEROSCLEROSIS}

N. Stoilov ${ }^{1}$, V. Boyadzhieva1. ' University Hospital "St. Ivan Rilski", Clinic of Rheumatology, Sofia, Bulgaria

Background: Antiphospholipid syndrome (APS) is a systemic autoimmune disease with unknown etiology and complex pathogenesis. Recent studies have shown that the pathogenesis of the atherosclerotic process is related to the inflammatory component of the immune response, as well as to elements of autoimmunity. A number of autoimmune rheumatic diseases, including rheumatoid arthritis (RA), SLE and APS, are characterized by accelerated atherosclerosis and therefore at increased risk of cardiovascular disease and mortality. The risk of early atherosclerosis in patients with autoimmune disease is determined by traditional risk factors, chronic generalized inflammation, and target-specific autoimmune processes. These processes are complemented by the chronic use of drugs, such as KS, which worsen BMI, lead to hyperglycemia and hyperlipidemia.

Objectives: The aim of this study is to investigate the incidence of atherosclerosis at selected typical sites in patients with APS.

Methods: We examined 139 patients devided in to three groups: APS, SLE and healty controls. We performed US examination of carotid atheries with IMT measeurement and Calcium score of coronary artheries and aorta. All the patients were tested for: CBC, lipid panel, liver probes, ESR, anti-CL, anti-b2GPI anti-Prothrombin antibodies and ANA screen and ANA-profile as well.

Results: With the Kruskall-Wallis analysis, we found a statistically significant difference in IMT between the study groups for left $(p=0.005)$ and right $(p=0.014)$ carotid arteries. Comparative analysis between the groups showed that pathological levels of IMT $(>900 \mu \mathrm{m})$ in the right carotid artery were the highest in patients with antiphospholipid syndrome 15 (26.3\%). In healthy controls, $3(7.3 \%)$ were identified and no cases were reported for patients with SLE. The data for the left carotid artery (LCCA) are similar. 15 (26.3\%) were registered for AFS, 4 for healthy controls $(9.2 \%)$, and no patients with pathological values for IMT were registered in the SLE group. We compared the results for intima-media ticking between groups with the Chi-square test, finding a statistically significant correlation for the two carotid arteries - IMT-RCCA $p=<0.001$ for IMT-LCCA $p=0.002$. Descriptive statistics show that the highest frequency of positive calcium score is characterized by the APS group $12(31.6 \%)$, in patients with SLE they are 3 $(15 \%)$, in healthy controls the case is $3 \%$.

In our study, the AFS group had the highest incidence of positive CaScore, and its maximum values were significantly higher than the other two groups. The maximum agatson score for patients with antiphospholipid syndrome is $908 \mathrm{HU}$ (Hounsfield Units), for SLE it is $2.1 \mathrm{HU}$, for healthy controls it is $233 \mathrm{HU}$.

With the Kruskall-Wallis statistical analysis we examined the relationship of calcium score with the individual groups. Aortic aortic valve score was negative in all subjects. We find statistically significant differences between the calcium score groups. Conclusion: The APS group is statistically significant in both pathologic IMT and higher plaque incidence compared to healthy controls and patients with SLE without the presence of anthophospholipid antibodies.

Based on the data obtained we can conclude that the antiphospholipid antibodies tested and their serum levels did not directly influence the values of the coronary artery and aortic Agatson score.

Disclosure of Interests: None declared

DOI: 10.1136/annrheumdis-2020-eular.6063

\section{AB0143 EXPRESSION OF HERV-E CLONE 4.1 GAG TRANSCRIPTS IN PERIPHERAL BLOOD MONONUCLEAR CELLS OF SYSTEMIC LUPUS ERYTHEMATOSUS PATIENTS}

R. Talotta ${ }^{1}$, A. Troldborg ${ }^{2}$, F. Atzeni ${ }^{1}$, M. J. Laska ${ }^{2} .{ }^{1}$ University of Messina, Clinical and Experimental Medicine, Rheumatology Unit, Messina, Italy; ${ }^{2}$ Aarhus University Hospital, Rheumatology, Aarhus, Denmark

Background: Human endogenous retrovirus (HERV)-E clone 4.1 gag transcripts have been detected in peripheral blood mononuclear cells (PBMCs) of systemic lupus erythematosus (SLE) patients and seem to be associated to autoantibody production. To date, no study aimed to investigate whether their expression in 